\title{
Micorriza arbuscular, Mucoromycotina y hongos septados oscuros en helechos y licófitas con distribución en México: una revisión global
}

\author{
Luis Alberto Lara-Pérez ${ }^{1 *}$, Ramón Zulueta-Rodríguez ${ }^{2} \&$ Antonio Andrade-Torres ${ }^{1}$ \\ 1. Instituto de Biotecnología y Ecología Aplicada, Universidad Veracruzana, Av. de las Culturas Veracruzanas No. 101, \\ Campus para la Cultura, las Artes y el Deporte, col. Emiliano Zapata, C. P. 91090, Xalapa, Veracruz, México; \\ CA-UVER-173; ingluislara@gmail.com, aandradet@gmail.com \\ 2. Laboratorio de Organismos Benéficos, Facultad de Ciencias Agrícolas, Universidad Veracruzana, Campus Xalapa. \\ Circuito Universitario Gonzalo Aguirre Beltrán s/n, Zona Universitaria, C. P. 91090, Xalapa, Veracruz, México; \\ rzulueta36@hotmail.com \\ * Correspondence
}

Recibido 10-VIII-2016. Corregido 21-IV-2017. Aceptado 19-V-2017.

\begin{abstract}
Arbuscular mycorrhizal, Mucoromycotina and dark septate fungi in ferns and lycophytes with distribution in Mexico: a global review. Ferns and lycophytes are a group of vascular plants of interest to understand the evolution of mycorrhizal interactions; their preservation is of relevance for their multiple ecological relations. The record of different taxonomic groups of fungi associated with ferns and lycophytes is fragmentary, and the criteria for it identification is inconsistent, which hinders the understanding and determination of mycorrhizal status. The aim of this study was to determine the percentage of the species of ferns and lycophytes with distribution in Mexico, and with information of fungal interactions. A checklist of the presence of arbuscular mycorrhizal fungi (AMF), Mucoromycotina and dark septate fungi (DSF) associated with ferns and lycophytes was integrated through an exhaustive global literature search. In this study, mycorrhizal species was considered by the presence of arbuscules to differentiate with hyphal, vesicular and coils colonization. The study gathered a checklist of mycorrhizal occurrences of 27 families, 61 genus and 137 species of ferns and lycophytes, which covers $13.4 \%$ of the species, $91 \%$ of the genus and $77 \%$ of the families distributed in Mexico. The $78.1 \%$ of the species showed colonization, $56.2 \%$ by AMF, $29.9 \%$ by DSF and $0.72 \%$ by Mucoromycotina fungi. From the total of the species, the higher presences of colonization were in terrestrial, epiphytic, saxicolous, and aquatic plants with $76.6 \%, 33.3 \%, 20 \%$, and $6.3 \%$, respectively. The families of ferns and lycophytes with the higher number of species colonized were Pteridaceae, Polypodiaceae, Aspleniaceae and Dryopteridaceae. The present study showed the widespread associations of AMF and DSF in ferns and lycophytes of Mexico. It is urgently needed to include ferns and lycophytes in studies focused on endomycorhizal interactions, since only 28 species (28\%) were studied in Mexican ecosystems. The majority of studies were focused on sporophytic face ( $80 \%)$. Nonetheless, to understand the role that plays the mycorrhiza in the establishment of ferns and lycophytes, it is necessary to include the gametophytic face in ecological, molecular and physiological experimental studies. This information is important to implement conservation strategies, because a considerable number of ferns and lycophytes species, depend on these mycorrhizal associations for their growth and survival. Rev. Biol. Trop. 65 (3): 1062-1081. Epub 2017 September 01.
\end{abstract}

Key words: arbuscular mycorrhizal fungi, dark septate fungi, Mucoromycotina, Lycophyta, Monilophyta, ferns.

Los helechos y licófitas son dos linajes de plantas vasculares evolutivamente divergentes que comparten características en su ciclo de vida con dos fases (gameto y esporofítica), y que tienen la capacidad de propagarse por esporas (Raghavan, 1992; Smith et al., 2006). Debido a estas características, se les agrupó con el nombre de Pteridophytas, helechos y plantas afines (Boullard, 1979; Tryon \& Tryon, 1982; Mickel, \& Smith, 2004), y recientemente como pteridobiontes (Tejero-Díez, Torres-Díaz, Mickel, Mehltreter, \& Krömer, 2009).

Se calcula que la riqueza de helechos y licófitas en el mundo es superior a las 12000 
especies, con un mayor número de especies en los trópicos (Moran, 2008). Las distintas formas de vida de este grupo de plantas pueden prosperar en ambientes terrestres, saxícolas, epífitos y acuáticos, por lo que difieren en sus estrategias de adquisición de nutrimentos, y tienen un amplio rango de hábitats que van desde bosques secos a los bosques templados (Mickel \& Smith, 2004).

Los helechos y licófitas juegan un papel importante en los diferentes ecosistemas, donde mantienen interacciones ecológicas con distintos organismos como hongos, lombrices, y artrópodos (Mehltreter, 2010). Una de las interacciones simbióticas más extendida en la mayoría de especies en este grupo de plantas es la denominada micorriza (hongo-raíz) (Brundrett, 2002). En la actualidad, se reconocen cuatro tipos de micorriza con base en su estructura y función: micorriza arbuscular, ectomicorriza, micorriza orquideoide y micorriza ericoide (van der Heijden, Martin, Selosse, \& Sanders, 2015). No obstante, en las plantas se pueden encontrar otras asociaciones con un grupo de hongos misceláneos como los hongos septados oscuros (HSO) y con miembros de los Mucoromycotina, que en algunas ocasiones, ambos son capaces de establecer interacciones mutualistas (Jumpponen, 2001; Bidartondo et al., 2011; Newsham, 2011; Field et al., 2015).

Los hongos micorrízicos arbusculares (HMA) son un grupo monofilético del phylum de los Glomeromycota con cerca de 270 taxa capaces de formar simbiosis mutualista con $72 \%$ de las plantas del mundo (Brundrett, 2009; Schüßler \& Walker, 2010). Las hifas extraradicales de los HMA funcionan como extensiones de la raíz que mejoran la absorción de nutrimentos y agua, y el hongo a cambio, recibe fotosintatos de su hospedero (Smith \& Read, 2008). Debido a la mejora nutricional, la planta tiene mayor tolerancia a estrés hídrico y patógenos, e influyen en la diversidad y en la adecuación de las especies vegetales (Smith \& Read, 2008). Además, permite el movimiento de nutrimentos carbonados interplanta debido a las conexiones hifales, y juega un papel importante en el ciclado de carbono, nitrógeno y fósforo (Kottke et al., 2008; Field et al., 2015; van der Heijden et al., 2015).

La micorriza arbuscular es una de las interacciones más antiguas que ha prevalecido desde hace unos 450 millones de años (Redecker, Kodner, \& Graham, 2000; Bonfante \& Selosse, 2010). Por lo que se sugiere, que fue la condición simbiótica ancestral que permitió que las plantas colonizaran el medio terrestre (Pirozynski \& Malloch, 1975; Bonfante \& Genre, 2008). Sin embargo, nuevas evidencias paleobotánicas y moleculares sugieren que los hongos pertenecientes a Mucoromycotina, debido a que son una rama más basal que los Glomeromycota, fueron los que facilitaron la colonización del medio terrestre por las plantas (James et al., 2006; Bidartondo et al., 2011; Strullu-Derrien et al., 2014).

En la actualidad, la principal interacción micorrízica que presentan los gametofitos y esporofitos de helechos y licófitas es con los HMA que forma la micorriza arbuscular (MA) (Boullard, 1979; Newman \& Reddell, 1987; Wang \& Qiu, 2006). La colonización de la MA se caracteriza por formar hifas en ovillos (coils) y estructuras de almacenamiento denominadas vesículas y una interface especializada para el intercambio de nutrimentos llamada arbúsculo (Smith \& Read, 2008). La presencia de los HMA en helechos y licófitas es funcional al incrementar la biomasa foliar (Cooper, 1976; Turnau, Anielska, \& Jurkiewicz, 2005). En ambientes naturales la micorriza provee una ventaja ecológica a helechos que crecen en sustratos pobres en nutrimientos (Kessler, Jonas, Strasberg, \& Lehnert, 2010), y recientemente, se demostró la primera evidencia directa del intercambio de carbono por nutrimentos durante la simbiosis micorrízica en Ophioglossum vulgatum L. (Field et al., 2015).

Algunas familias como Dryopteridaceae, Equisetaceae, Hymenophyllaceae, Lycopodiaceae y Polypodiaceae (grammítidos) forman asociaciones con HSO que se distinguen por ser dematiáceos a hialinos, con hifas septadas, presencia ocasional de microesclerocios y células moniliformes (Fernández, Messuti, \& Fontenla, 2008; Lehnert et al., 2009; Kessler 
et al., 2009). La presencia de los HSO en las diferentes familias de helechos y licófitas podría sugerir que estos hongos tienen un papel ecológico importante para el crecimiento y desarrollo de las especies.

El estudio de los hongos Mucoromycotina está iniciado y por ahora se han registrado en las familias Lycopodiaceae y Ophioglosaceae (Rimington, Pressel, Duckett, \& Bidartondo, 2015). Estos hongos están presentes por hifas intracelulares, ovillos de vida corta y vesículas difíciles de distinguir de los Glomeromycota cuando se analiza con microscopio compuesto (Bidartondo et al., 2011; Rimington et al., 2015).

Dentro de los países del trópico, México se distingue por tener una alta riqueza de helechos y licófitas (Mickel \& Smith, 2004; Moran, 2008). De acuerdo con la clasificación de Smith et al. (2006) y Christenhusz, Zhang y Schneider (2011), alberga 32 familias con 902 especies de helechos y tres familias con 106 especies de licófitas, para un total de 1008 especies, de las cuales el $10.5 \%$ son endémicas (Mickel \& Smith, 2004). La heterogeneidad de ecosistemas en México, que van desde el nivel del mar hasta la alta montaña, es un escenario ideal para responder preguntas ecológicas relacionadas con las interacciones micorrízicas en helechos y licófitas. Los estudios relativos a la interacción planta-hongo micorrizógenos incluyen especies de plantas en ecosistemas naturales y en sistemas de producción agrícola (Alarcón, Hernández-Cuevas, Ferrera-Cerrato, \& Franco-Ramírez, 2012; Montaño et al., 2012) y pocos son los que incluyen a helechos y licófitas (Lara-Pérez et al., 2015), a pesar de que muchas especies dependen de la interacción micorrízica para reclutar individuos en las poblaciones naturales (Leake, 1994; Winther \& Friedman, 2008; Field et al., 2015). El conocimiento de la identidad y funcionalidad de los hongos asociados a plantas de ramas basales como las Licófitas y Monilofitas es clave para entender el papel de los hongos micorrízicos en la evolución de las plantas en la tierra (Merckx \& Bidartondo, 2008). Asimismo, la afinidad de los diferentes linajes de hongos en los sustratos donde se desarrollan los helechos y licófitas podría permitir tomar decisiones para un manejo adecuado para su propagación y conservación, con un impacto en la restauración de los ecosistemas. No obstante, el registro de los diferentes grupos taxonómicos de hongos asociados a helechos y licófitas, se encuentra fragmentado en diferentes temas de investigación, y para el caso de los HMA, los criterios para su diagnóstico son inconsistentes, lo que dificulta el entendimiento y la determinación del estatus micorrízico. Debido a que la riqueza de los helechos y licófitas tienen una amplia distribución, y se comparten en diferentes regiones y continentes, los objetivos del presente trabajo fueron: 1) estimar el porcentaje de helechos y licófitas registrados para México en los que se han abordado estudios de interacciones con HMA, HSO, Mucoromycotina, a través de una compilación de literatura mundial, y 2) realizar un diagnóstico del estatus micorrízico arbuscular, la presencia de HSO y Mucoromycotina en helechos y licófitas con distribución en México, y proveer un listado detallado de la ocurrencia de estas interacciones endomicorrízicas.

\section{MATERIALES Y MÉTODOS}

\section{Estrategia de la búsqueda de literatura:} Se realizó una revisión de la literatura mundial para compilar el estado del conocimiento de hongos micorrízicos arbusculares, hongos septados oscuros y Mucoromycotina en helechos y licopodios distribuidos en México, de acuerdo con Mickel y Smith (2004). La revisión consistió en una búsqueda exhaustiva de información en tres diferentes bases de datos: ISI Web of Knowledge, Scopus y Google Scholar. Los términos utilizados fueron: arbuscular, vesicular, mycorrhiza, dark septate fungi, Mucoromycotina y endophyte en combinación con las entradas: Pteridophyta, fern, fern allie, gametophyte, sporophyte y cada uno de los géneros de helechos incluidos en Smith et al. (2006) más los géneros de licófitas incluidos en las familias Lycopodiaceae, Selaginellaceae, e Isoëtaceae (Christenhusz et al., 2011). En la revisión se tomaron en cuenta 
los trabajos que evaluaron la asociación de hongos micorrízicos por métodos de clareo y tinción, inoculación en condiciones controladas y detección por métodos moleculares, en la fase gametofítica y esporofítica. En la base de datos se incluyeron las siguientes clasificaciones: a) micorrízico (cuando se registró presencia de arbúsculos), b) colonización por hifas y/o vesículas, c) ausente de colonización, d) presencia de hongos septados oscuros y e) hongos Mucoromycotina. La base de datos se empezó a generar en enero de 2012 y los últimos registros se realizaron en diciembre de 2015. Los helechos y licófitas tienen una amplia distribución biogeográfica y pueden habitar en diferentes ecosistemas, por tales razones, para determinar si una especie es consistentemente micorrízica, se consideró cada trabajo como una muestra independiente para cubrir diferentes ecosistemas, estaciones, posibles estados fenológicos y disturbios. El sustrato de los helechos se categorizó como acuático, terrestre, epífito y saxícola. Finalmente, las especies y familias se ordenaron de acuerdo a la clasificación de helechos y licófitas propuesta por Smith et al. (2006) y Christenhusz et al. (2011). Las especies de helechos y licófitas registradas para México en Mickel y Smith (2004) se reordenaron de acuerdo a Smith et al. (2006), para estimar el porcentaje de especies con las que se cuenta con información de las interacciones micorrízicas.

\section{RESULTADOS}

De la revisión mundial total se obtuvo información de 1208 especies, de las cuales 137 son especies de helechos y licopodios con distribución en México, resultado de 59 trabajos de investigación. De la información reunida, sobre la interacción de hongos micorrízicos con helechos y licófitas, el $20 \%$ (28 especies) proviene de estudios realizados en México y el resto de diferentes países con los que se comparten las especies de ambos grupos. La revisión contiene 234 muestras de helechos y licófitas que incluyen 27 familias, 61 géneros y 137 especies (Cuadro 1). Del total de las

CUADRO 1

Listado de la presencia de hongos micorrízicos arbusculares, Mucoromycotina y hongos septados oscuros en helechos y licófitas con distribución en México

TABLE 1

Checklist of the occurrence of arbuscular mycorrhizal, Mucoromycotina, and dark septate fungi in ferns and lycophytes with distribution in Mexico

\begin{tabular}{|c|c|c|c|c|c|}
\hline Familia/Especie & Sustrato & Colonización & HMA & $\mathrm{HSO}$ & Referencias \\
\hline \multicolumn{6}{|l|}{ Anemiaceae } \\
\hline Anemia hirsuta (L.) Sw. & S & + & A & - & 35 \\
\hline Anemia phyllitidis (L.) Sw. & $\mathrm{T}$ & + & HVC & - & 1 \\
\hline Anemia phyllitidis & $\mathrm{T}$ & + & AV & + & 59 \\
\hline \multicolumn{6}{|l|}{ Aspleniaceae } \\
\hline Asplenium achilleifolium (M. Martens \& Galeotti) Liebm. & $\mathrm{E}$ & + & - & + & 59 \\
\hline Asplenium auriculatum $\mathrm{Sw}$. & $\mathrm{E}$ & - & - & - & 30 \\
\hline Asplenium auriculatum & $\mathrm{E}$ & + & - & + & 59 \\
\hline Asplenium auritum $\mathrm{Sw}$. & $\mathrm{E}$ & - & - & - & 30 \\
\hline Asplenium dalhousiae Hook. & $\mathrm{T}$ & + & A & - & 17 \\
\hline Asplenium fragrans Sw. & $\mathrm{E}$ & - & - & - & 59 \\
\hline Asplenium harpeodes Kunze & $\mathrm{T}$ & - & - & - & 19 \\
\hline Asplenium laetum $\mathrm{Sw}$. & $\mathrm{T}$ & + & A & - & 19 \\
\hline Asplenium miradorense Liebm. & $\mathrm{T}$ & - & - & - & 26 \\
\hline Asplenium monanthes L. & $\mathrm{T}$ & - & - & - & 35 \\
\hline
\end{tabular}


CUADRO 1 (Continuación) / TABLE 1 (Continued)

\begin{tabular}{|c|c|c|c|c|c|}
\hline Familia/Especie & Sustrato & Colonización & HMA & HSO & Referencias \\
\hline Asplenium praemorsum $\mathrm{Sw}$. & E & - & - & - & 59 \\
\hline Asplenium rutaceum (Willd.) Mett. & $\mathrm{T}$ & - & - & - & 19 \\
\hline Asplenium serra Langsd. \& Fisch. & $\mathrm{T}$ & - & - & - & 19 \\
\hline Asplenium serra & $\mathrm{E}$ & + & - & + & 59 \\
\hline Asplenium trichomanes $\mathrm{L}$. & $\mathrm{T}$ & - & - & - & 4 \\
\hline Asplenium trichomanes & $\mathrm{T}$ & + & $\mathrm{A}$ & - & 16 \\
\hline Asplenium trichomanes & $\mathrm{T}$ & + & A & - & 17 \\
\hline Asplenium trichomanes & $\mathrm{S}$ & - & - & - & 35 \\
\hline \multicolumn{6}{|l|}{ Blechnaceae } \\
\hline Blechnum appendiculatum Willd. & $\mathrm{T}$ & + & HVA & - & 3 \\
\hline Blechnum appendiculatum & $\mathrm{T}$ & + & $\mathrm{HV}$ & + & 26 \\
\hline Blechnum divergens (Kunze) Mett. & $\mathrm{T}$ & + & $\mathrm{A}$ & - & 19 \\
\hline Blechnum occidentale L. & $\mathrm{T}$ & + & A & - & 15 \\
\hline Blechnum penna-marina (Poir.) Kuhn & $\mathrm{T}$ & + & ND & - & 7 \\
\hline Blechnum penna-marina & $\mathrm{T}$ & + & ND & - & 10 \\
\hline Blechnum penna-marina & $\mathrm{T}$ & + & A & + & 14 \\
\hline Blechnum penna-marina & A & - & - & - & 27 \\
\hline Blechnum schiedeanum (Schltdl. ex C. Presl) Hieron. & $\mathrm{T}$ & + & HVA & + & 26 \\
\hline \multicolumn{6}{|l|}{ Cyatheaceae } \\
\hline Alsophila firma (Baker) D. S. Conant & $\mathrm{T}$ & + & A & + & 25 \\
\hline Alsophila firma & $\mathrm{T}$ & + & HVA & + & 26 \\
\hline Cyathea bicrenata Liebm. & $\mathrm{T}$ & + & HVA & - & 26 \\
\hline Cyathea divergens var. tuerckheimii (Maxon) R. M. Tryon & $\mathrm{T}$ & + & HVA & + & 26 \\
\hline Cyathea fulva (M. Martens \& Galeotti) Fée & $\mathrm{T}$ & + & A & - & 24 \\
\hline \multicolumn{6}{|l|}{ Dennstaedtiaceae } \\
\hline Dennstaedtia auriculata H. Navarrete \& B. Øllgaard & $\mathrm{T}$ & + & A & - & 19 \\
\hline Histiopteris incisa (Thunb.) J. Sm. & $\mathrm{T}$ & + & ND & - & 10 \\
\hline Histiopteris incisa & $\mathrm{T}$ & + & A & - & 58 \\
\hline Pteridium aquilinum (L.) Kuhn in v. d. Decken & $\mathrm{T}$ & + & A & - & 4 \\
\hline Pteridium aquilinum & $\mathrm{T}$ & + & ND & - & 10 \\
\hline Pteridium aquilinum & $\mathrm{T}$ & + & A & - & 16 \\
\hline Pteridium aquilinum & $\mathrm{T}$ & + & A & - & 23 \\
\hline Pteridium aquilinum & $\mathrm{T}$ & + & $\mathrm{VH}$ & - & 34 \\
\hline Pteridium aquilinum & $\mathrm{T}$ & - & - & - & 35 \\
\hline Pteridium aquilinum & $\mathrm{T}$ & + & A & - & 36 \\
\hline Pteridium aquilinum & $\mathrm{T}$ & + & HAVC & - & 38 \\
\hline Pteridium aquilinum & $\mathrm{T}$ & + & $\mathrm{CV}$ & - & 57 \\
\hline Pteridium aquilinum var. latiusculum (Desv.) Underw. ex Heller & $\mathrm{T}$ & + & VA & - & 28 \\
\hline \multicolumn{6}{|l|}{ Dicksoniaceae } \\
\hline Dicksonia sellowiana Hook. & $\mathrm{T}$ & + & A & - & 19 \\
\hline Lophosoria quadripinnata (J. F. Gmel.) C. Chr. & $\mathrm{T}$ & + & ND & - & 8 \\
\hline Lophosoria quadripinnata & $\mathrm{T}$ & + & A & + & 14 \\
\hline Lophosoria quadripinnata & $\mathrm{T}$ & + & A & - & 19 \\
\hline \multicolumn{6}{|l|}{ Dryopteridaceae } \\
\hline Arachniodes denticulata (Sw.) Ching & $\mathrm{T}$ & + & - & $?$ & 29 \\
\hline Ctenitis microchlaena (Fée) Stolze & $\mathrm{T}$ & + & A & - & 19 \\
\hline Elaphoglossum erinaceum (Fée) T. Moore & $\mathrm{E}$ & + & - & + & 59 \\
\hline Elaphoglossum peltatum (Sw.) Urb. & $\mathrm{E}$ & - & - & - & 30 \\
\hline
\end{tabular}


CUADRO 1 (Continuación) / TABLE 1 (Continued)

\begin{tabular}{|c|c|c|c|c|c|}
\hline Familia/Especie & Sustrato & Colonización & HMA & HSO & Referencias \\
\hline Elaphoglossum setigerum (Sodiro) Diels & E & - & - & - & 59 \\
\hline Elaphoglossum squarrosum (Klotzsch) T. Moore & $\mathrm{T}$ & + & A & - & 29 \\
\hline Elaphoglossum squarrosum & $\mathrm{T}$ & + & A & - & 29 \\
\hline Polystichum acrostichoides (Michx.) Schott & $\mathrm{T}$ & + & A & - & 4 \\
\hline Polystichum acrostichoides & $\mathrm{T}$ & + & ND & - & 53 \\
\hline Polystichum muricatum (L.) Fée & $\mathrm{T}$ & + & A & - & 19 \\
\hline Polystichum ordinatum (Kunze) Liebm. & $\mathrm{T}$ & + & $\mathrm{H}$ & - & 26 \\
\hline Polystichum platyphyllum (Willd.) C. Presl & $\mathrm{T}$ & + & A & - & 29 \\
\hline \multicolumn{6}{|l|}{ Equisetaceae } \\
\hline Equisetum hyemale var. affine (Engelm.) A. A. Eaton & $\mathrm{T}$ & - & - & - & 4 \\
\hline Equisetum laevigatum A. Braun & $\mathrm{T}$ & + & HA & - & 13 \\
\hline Equisetum laevigatum & $\mathrm{T}$ & + & $\mathrm{H}$ & - & 13 \\
\hline \multicolumn{6}{|l|}{ Gleicheniaceae } \\
\hline Dicranopteris flexuosa (Schrad.) Underw. & $\mathrm{T}$ & + & ND & - & 48 \\
\hline Diplopterygium bancroftii (Hook.) A. R. Sm. & $\mathrm{T}$ & + & AV & + & 59 \\
\hline Sticherus palmatus (W. Schaffn. ex E. Fourn.) Copel. & $\mathrm{T}$ & + & HVA & + & 26 \\
\hline Sticherus underwoodianus (Maxon) Nakai & $\mathrm{T}$ & + & A & + & 59 \\
\hline \multicolumn{6}{|l|}{ Hymenophyllaceae } \\
\hline Hymenophyllum fucoides (Sw.) Sw. & $\mathrm{E}$ & + & - & + & 29 \\
\hline Hymenophyllum hirsutum (L.) Sw. & $\mathrm{E}$ & + & Asc & - & 46 \\
\hline Hymenophyllum microcarpum Desv. & $\mathrm{E}$ & - & - & - & 30 \\
\hline Hymenophyllum polyanthos (Sw.) Sw. & $\mathrm{E}$ & + & - & + & 29 \\
\hline Hymenophyllum tunbrigense (L.) Sm. & $\mathrm{S}$ & - & - & - & 35 \\
\hline Trichomanes diversifrons (Bory) Mett. ex Sadeb. & $\mathrm{T}$ & + & A & - & 19 \\
\hline Trichomanes pinnatum Hedw. & $\mathrm{T}$ & + & A & - & 19 \\
\hline \multicolumn{6}{|l|}{ Lomariopsidaceae } \\
\hline Nephrolepis biserrata (Sw.) Schott & $\mathrm{E}$ & + & $\mathrm{HCV}$ & - & 40 \\
\hline Nephrolepis cordifolia (L.) C. Presl & $\mathrm{T}$ & + & A & - & 15 \\
\hline Nephrolepis cordifolia & $\mathrm{T}$ & + & - & + & 36 \\
\hline Nephrolepis cordifolia & $\mathrm{T}$ & + & HAVC & + & 38 \\
\hline Nephrolepis exaltata (L.) Schott & $\mathrm{T}$ & + & A & - & 15 \\
\hline Nephrolepis exaltata & $\mathrm{T}$ & + & HA & + & 37 \\
\hline Nephrolepis exaltata & $\mathrm{T}$ & + & HAVC & - & 38 \\
\hline \multicolumn{6}{|l|}{ Lycopodiaceae } \\
\hline Huperzia crassa (Humb. \&Bonpl. ex Willd.) Rothm. & $\mathrm{T}$ & + & A & - & 55 \\
\hline Huperzia serrata (Thunb. ex Murray) Trevis. & $\mathrm{T}$ & - & - & - & 58 \\
\hline Lycopodiella cernua (L.) Pic. Serm. & $\mathrm{T}$ & - & - & - & 36 \\
\hline Lycopodiella cernua & $\mathrm{T}$ & + & A & - & 43 \\
\hline Lycopodium clavatum $\mathrm{L}$. & $\mathrm{T}$ & - & - & - & 5 \\
\hline Lycopodium clavatum & $\mathrm{T}$ & + & HAVC & - & 38 \\
\hline Lycopodium clavatum & $\mathrm{T}$ & + & Asc & - & 47 \\
\hline Lycopodium clavatum & $\mathrm{T}$ & + & - & + & 50 \\
\hline Lycopodium thyoides Humb. \& Bonpl. & $\mathrm{T}$ & - & - & - & 5 \\
\hline Lycopodium thyoides & $\mathrm{T}$ & + & - & + & 59 \\
\hline \multicolumn{6}{|l|}{ Macrothelypteris } \\
\hline Macrothelypteris torresiana (Gaudich.) Ching & $\mathrm{T}$ & + & HVA & - & 36 \\
\hline Macrothelypteris torresiana & $\mathrm{T}$ & + & HVAC & + & 39 \\
\hline
\end{tabular}


CUADRO 1 (Continuación) / TABLE 1 (Continued)

\begin{tabular}{|c|c|c|c|c|c|}
\hline Familia/Especie & Sustrato & Colonización & HMA & $\mathrm{HSO}$ & Referencias \\
\hline \multicolumn{6}{|l|}{ Marattiaceae } \\
\hline Danaea elliptica $\mathrm{Sm}$. & $\mathrm{T}$ & + & A & - & 19 \\
\hline \multicolumn{6}{|l|}{ Marsileaceae } \\
\hline Marsilea vestita Hook. \& Grev. & $\mathrm{A}$ & + & HVA & - & 2 \\
\hline Marsilea vestita & A & + & HVAC & + & 39 \\
\hline Marsilea polycarpa Hook. \& Grev. & A & + & - & + & 49 \\
\hline Marsilea vestita & A & - & - & - & 49 \\
\hline \multicolumn{6}{|l|}{ Metaxyaceae } \\
\hline Metaxya rostrata (Kunth) C. Presl & $\mathrm{T}$ & + & A & - & 19 \\
\hline \multicolumn{6}{|l|}{ Ophioglossaceae } \\
\hline Botrychium virginianum (L.) Sw. & $\mathrm{T}$ & + & A & - & 4 \\
\hline Botrychium virginianum & $\mathrm{T}$ & + & A & - & 19 \\
\hline Botrychium virginianum & $\mathrm{T}$ & + & A & - & 21 \\
\hline Botrychium virginianum & $\mathrm{T}$ & + & A & - & 22 \\
\hline Botrychium virginianum & $\mathrm{T}$ & + & HV & - & 34 \\
\hline Botrychium virginianum & $\mathrm{T}$ & + & A & - & 53 \\
\hline Ophioglossum reticulatum L. & $\mathrm{T}$ & + & AV & + & 38 \\
\hline Ophioglossum reticulatum & $\mathrm{T}$ & + & $\mathrm{HA}$ & - & 45 \\
\hline Ophioglossum reticulatum & $\mathrm{T}$ & - & - & - & 58 \\
\hline Ophioglossum vulgatum $\mathrm{L}$. & $\mathrm{T}$ & + & A & - & 16 \\
\hline Ophioglossum vulgatum & $\mathrm{T}$ & + & A & - & 43 \\
\hline Ophioglossum vulgatum & $\mathrm{T}$ & + & $\mathrm{CV}$ & - & 57 \\
\hline \multicolumn{6}{|l|}{ Osmundaceae } \\
\hline Osmunda cinnamomea L. & $\mathrm{T}$ & + & A & - & 4 \\
\hline Osmunda cinnamomea & $\mathrm{T}$ & + & CVA & - & 9 \\
\hline Osmunda cinnamomea & $\mathrm{T}$ & + & A & - & 23 \\
\hline \multicolumn{6}{|l|}{ Polypodiaceae } \\
\hline Campyloneurum amphostenon (Kunze ex Klotzsch) Fée & $\mathrm{T}$ & - & - & - & 19 \\
\hline Campyloneurum amphostenon & $\mathrm{E}$ & - & - & - & 29 \\
\hline Campyloneurum amphostenon & $\mathrm{E}$ & - & - & - & 59 \\
\hline Campyloneurum angustifolium (Sw.) Fée & $\mathrm{E}$ & + & - & + & 59 \\
\hline Campyloneurum costatum (Kunze) C. Presl & $\mathrm{E}$ & + & HA & - & 26 \\
\hline Campyloneurum tenuipes Maxon & $\mathrm{E}$ & + & - & + & 59 \\
\hline Cochlidium serrulatum (Sw.) L. E. Bishop & $\mathrm{E}$ & + & - & + & 29 \\
\hline Enterosora parietina (Klotzsch) L. E. Bishop & $\mathrm{E}$ & - & - & - & 29 \\
\hline Lellingeria prionodes (Mickel \& Beitel) A. R. Sm. \& R. C. Moran & $\mathrm{E}$ & + & - & + & 59 \\
\hline Melpomene firma (J. Sm.) A. R. Sm. \& R. C. Moran & $\mathrm{E}$ & + & - & + & 29 \\
\hline Melpomene flabelliformis (Poir.) A. R. Sm. \& R. C. Moran & $\mathrm{S}$ & - & - & - & 35 \\
\hline Melpomene moniliformis (Lag. ex Sw.) A. R. Sm. \& R. C. Moran & $\mathrm{T}$ & + & - & + & 29 \\
\hline Microgramma reptans (Cav.) A. R. Sm. & $\mathrm{E}$ & - & - & - & 30 \\
\hline Niphidium crassifolium (L.) Lellinger & $\mathrm{E}$ & + & HVA & - & 26 \\
\hline Niphidium crassifolium & $\mathrm{T}$ & + & - & + & 59 \\
\hline Pecluma dispersa (A. M. Evans) M. G. Price & $\mathrm{S}$ & - & - & - & 26 \\
\hline Phlebodium areolatum (Humb. \& Bonpl. ex Willd.) J. Sm. & $\mathrm{T}$ & - & - & - & 15 \\
\hline Phlebodium areolatum & $\mathrm{E}$ & + & $\mathrm{H}$ & - & 26 \\
\hline Phlebodium areolatum & $\mathrm{E}$ & + & $\mathrm{H}$ & - & 26 \\
\hline Pleopeltis crassinervata (Fée) T. Moore & $\mathrm{S}$ & + & $\mathrm{HV}$ & + & 26 \\
\hline Pleopeltis mexicana (Fée) Mickel \& Beitel & $\mathrm{S}$ & - & - & - & 35 \\
\hline
\end{tabular}


CUADRO 1 (Continuación) / TABLE 1 (Continued)

\begin{tabular}{|c|c|c|c|c|c|}
\hline Familia/Especie & Sustrato & Colonización & HMA & HSO & Referencias \\
\hline Pleopeltis mexicana & E & - & - & - & 59 \\
\hline Pleopeltis polypodioides & $\mathrm{E}$ & - & - & - & 59 \\
\hline Polypodium furfuraceum Schltdl. \& Cham. & $\mathrm{E}$ & - & - & - & 30 \\
\hline Polypodium lepidotrichum (Fée) Maxon & $\mathrm{T}$ & + & HA & - & 26 \\
\hline Polypodium plebeium Schltdl. \& Cham. & $\mathrm{E}$ & - & - & - & 59 \\
\hline Polypodium polypodioides (L.) Watt (Pleopeltis polypodioides) & $\mathrm{S}$ & - & - & - & 35 \\
\hline Polypodium subpetiolatum Hook. & $\mathrm{E}$ & - & - & - & 59 \\
\hline Terpsichore semihirsuta (Klotzsch) A. R. Sm. & $\mathrm{T}$ & + & - & + & 29 \\
\hline \multicolumn{6}{|l|}{ Psilotaceae } \\
\hline Psilotum complanatum Sw. & $\mathrm{E}$ & - & - & - & 15 \\
\hline Psilotum nudum (L.) P. Beauv. & $\mathrm{T}$ & + & A & - & 15 \\
\hline Psilotum nudum & $\mathrm{T}$ & + & $\mathrm{C}$ & - & 38 \\
\hline Psilotum nudum & $\mathrm{E}$ & - & - & - & 40 \\
\hline Psilotum nudum & $\mathrm{T}$ & + & A & - & 43 \\
\hline Psilotum nudum & $\mathrm{T}$ & + & A & - & 54 \\
\hline \multicolumn{6}{|l|}{ Pteridaceae } \\
\hline Adiantum andicola Liebm. & $\mathrm{T}$ & + & HVA & + & 26 \\
\hline Adiantum capillus-veneris L. & $\mathrm{T}$ & + & HV & - & 3 \\
\hline Adiantum capillus-veneris & $\mathrm{T}$ & - & - & - & 11 \\
\hline Adiantum capillus-veneris & $\mathrm{S}$ & + & A & - & 15 \\
\hline Adiantum capillus-veneris & $\mathrm{T}$ & + & A & - & 16 \\
\hline Adiantum capillus-veneris & $\mathrm{T}$ & + & A & - & 17 \\
\hline Adiantum capillus-veneris & $\mathrm{S}$ & - & - & - & 35 \\
\hline Adiantum capillus-veneris & $\mathrm{T}$ & + & $\mathrm{HV}$ & - & 44 \\
\hline Adiantum capillus-veneris & $\mathrm{T}$ & - & - & - & 56 \\
\hline Adiantum concinnum Humb. \& Bonpl. ex Willd. & $\mathrm{T}$ & + & $\mathrm{HV}$ & - & 26 \\
\hline Adiantum latifolium Lam. & $\mathrm{T}$ & - & - & - & 18 \\
\hline Adiantum latifolium & $\mathrm{E}$ & - & - & - & 40 \\
\hline Adiantum lunulatum Burm. & $\mathrm{T}$ & + & $\mathrm{HV}$ & - & 20 \\
\hline Adiantum lunulatum & $\mathrm{T}$ & + & HAVC & + & 38 \\
\hline Adiantum poiretii Wikstr. & $\mathrm{T}$ & - & - & - & 35 \\
\hline Adiantum raddianum $\mathrm{C}$. Presl & $\mathrm{T}$ & + & HVA & + & 36 \\
\hline Adiantum raddianum & $\mathrm{T}$ & + & HAVC & - & 38 \\
\hline Adiantum raddianum & $\mathrm{T}$ & + & HA & - & 44 \\
\hline Adiantum raddianum & $\mathrm{T}$ & + & A & - & 52 \\
\hline Adiantum trapeziforme $\mathrm{L}$. & $\mathrm{T}$ & + & HV & + & 26 \\
\hline Anogramma leptophylla (L.) Link ${ }^{*}$ & $\mathrm{~T}$ & + & A & - & 43 \\
\hline Anogramma leptophylla & $\mathrm{T}$ & + & - & - & 6 \\
\hline Ceratopteris thalictroides (L.) Brongn. & A & - & - & - & 15 \\
\hline Ceratopteris thalictroides & A & - & - & - & 28 \\
\hline Ceratopteris thalictroides & $\mathrm{T}$ & + & HVAC & + & 39 \\
\hline Cheilanthes bonariensis (Willd.) Proctor & $\mathrm{T}$ & + & HVA & - & 3 \\
\hline Cheilanthes farinosa (Forssk.) Kaulf. & $\mathrm{T}$ & + & A & - & 17 \\
\hline Cheilanthes farinosa & $\mathrm{T}$ & + & HAV & - & 34 \\
\hline Cheilanthes feei T. Moore & $\mathrm{S}$ & + & HVA & - & 41 \\
\hline Cheilanthes myriophylla Desv. & $\mathrm{T}$ & + & HV & - & 3 \\
\hline Doryopteris concolor (Langsd. \& Fisch.) Kuhn & $\mathrm{T}$ & - & - & - & 35 \\
\hline
\end{tabular}


CUADRO 1 (Continuación) / TABLE 1 (Continued)

\begin{tabular}{|c|c|c|c|c|c|}
\hline Familia/Especie & Sustrato & Colonización & HMA & HSO & Referencias \\
\hline Doryopteris concolor & $\mathrm{T}$ & + & HA & + & 36 \\
\hline Dryopteris filix-mas (L.) Schott & $\mathrm{T}$ & + & A & - & 16 \\
\hline Dryopteris muenchii A. R. Sm. & $\mathrm{T}$ & + & $\mathrm{A}$ & - & 42 \\
\hline Dryopteris wallichiana (Spreng.) Hyl. & $\mathrm{T}$ & + & A & - & 15 \\
\hline Pellaea ternifolia (Cav.) Link & $\mathrm{T}$ & + & A & - & 15 \\
\hline Pellaea ternifolia subsp. ternifolia & $\mathrm{T}$ & + & $\mathrm{A}$ & - & 15 \\
\hline Pityrogramma calomelanos (L.) Link & $\mathrm{T}$ & + & $\mathrm{A}$ & - & 18 \\
\hline Pityrogramma calomelanos & $\mathrm{T}$ & + & $\mathrm{HV}$ & - & 20 \\
\hline Pityrogramma calomelanos & $\mathrm{T}$ & + & HVA & - & 36 \\
\hline $\begin{array}{l}\text { Polytaenium feei (W. Schaffn. ex Fée) } \\
\text { Maxon (Antrophyum lanceolatum) }\end{array}$ & $\mathrm{E}$ & - & - & - & 30 \\
\hline Pteris biaurita $\mathrm{L}$. & $\mathrm{T}$ & + & VAH & - & 34 \\
\hline Pteris biaurita & $\mathrm{T}$ & + & HVAC & + & 39 \\
\hline Pteris biaurita & $\mathrm{T}$ & + & HA & - & 44 \\
\hline Pteris cretica $\mathrm{L}$. & $\mathrm{T}$ & + & A & - & 15 \\
\hline Pteris cretica & $\mathrm{T}$ & - & - & - & 16 \\
\hline Pteris cretica & $\mathrm{T}$ & + & A & - & 17 \\
\hline Pteris cretica & $\mathrm{T}$ & + & A & - & 28 \\
\hline Pteris cretica & $\mathrm{S}$ & + & A & - & 35 \\
\hline Pteris cretica & $\mathrm{T}$ & + & HAVC & - & 38 \\
\hline Pteris muricata Hook. & $\mathrm{T}$ & + & A & - & 19 \\
\hline Pteris podophylla $\mathrm{Sw}$. & $\mathrm{T}$ & + & A & + & 19 \\
\hline Pteris quadriaurita Sw. & $\mathrm{T}$ & + & VAH & - & 34 \\
\hline Pteris vittata $\mathrm{L}$. & $\mathrm{T}$ & + & A & - & 15 \\
\hline Pteris vittata & $\mathrm{T}$ & + & $\mathrm{HV}$ & - & 20 \\
\hline Pteris vittata & $\mathrm{T}$ & + & AVC & - & 31 \\
\hline Pteris vittata & $\mathrm{T}$ & + & A & - & 32 \\
\hline Pteris vittata & $\mathrm{T}$ & + & AVH & - & 33 \\
\hline Pteris vittata & $\mathrm{T}$ & + & VAH & - & 34 \\
\hline Pteris vittata & $\mathrm{S}$ & + & A & - & 35 \\
\hline Pteris vittata & $\mathrm{T}$ & + & HAV & - & 38 \\
\hline Pteris vittata & $\mathrm{T}$ & + & A & - & 51 \\
\hline Pteris vittata & $\mathrm{T}$ & + & $\mathrm{CV}$ & - & 57 \\
\hline Pteris vittata & $\mathrm{T}$ & + & A & - & 58 \\
\hline \multicolumn{6}{|l|}{ Saccolomataceae } \\
\hline Saccoloma elegans Kaulf. & $\mathrm{T}$ & + & A & - & 19 \\
\hline Saccoloma inaequale (Kunze) Mett. & $\mathrm{T}$ & + & A & + & 19 \\
\hline \multicolumn{6}{|l|}{ Salviniaceae } \\
\hline Salvinia auriculata Aubl. & A & + & - & + & 12 \\
\hline Salvinia minima Baker & A & + & - & + & 12 \\
\hline Azolla filiculoides Lam. & A & - & - & - & 15 \\
\hline \multicolumn{6}{|l|}{ Schizaeaceae } \\
\hline Schizaea elegans (Vahl) Sw. & $\mathrm{T}$ & + & A & - & 19 \\
\hline \multicolumn{6}{|l|}{ Selaginellaceae } \\
\hline Selaginella apoda (L.) Spring & $\mathrm{T}$ & + & A & - & 23 \\
\hline Selaginella finitima Mickel \& Beitel & $\mathrm{T}$ & + & $\mathrm{HV}$ & + & 26 \\
\hline Selaginella martensii Spring & $\mathrm{T}$ & + & $\mathrm{H}$ & - & 26 \\
\hline
\end{tabular}


CUADRO 1 (Continuación) / TABLE 1 (Continued)

\begin{tabular}{|c|c|c|c|c|c|}
\hline Familia/Especie & Sustrato & Colonización & HMA & HSO & Referencias \\
\hline \multicolumn{6}{|l|}{ Thelypteridaceae } \\
\hline Thelypteris caucaensis (Hieron.) Alston & $\mathrm{T}$ & + & A & - & 19 \\
\hline Thelypteris interrupta (Willd.) K. Iwats. & $\mathrm{T}$ & - & - & - & 15 \\
\hline Thelypteris rudis (Kunze) Proctor & $\mathrm{T}$ & + & A & - & 19 \\
\hline \multicolumn{6}{|l|}{ Woodsiaceae } \\
\hline Cystopteris fragilis (L.) Bernh. & $\mathrm{T}$ & - & - & - & 4 \\
\hline Cystopteris fragilis & $\mathrm{T}$ & - & - & - & 19 \\
\hline Cystopteris fragilis & S & + & A & - & 35 \\
\hline Diplazium expansum Willd. & $\mathrm{T}$ & + & A & - & 19 \\
\hline Diplazium hians Kunze ex Klotzsch & $\mathrm{T}$ & + & A & - & 19 \\
\hline
\end{tabular}

$*$ Especies asociadas a hogos Mucoromycotina; Asco = Ascomycota; $\mathrm{ND}=$ Criterio de diagnóstico no determinado; $\mathrm{T}=$ Terrestre; $\mathrm{S}=$ Saxícola; $\mathrm{E}=$ Epífita; $\mathrm{A}=$ Acuática.

Andrade et al., 2000, Bajwa et al., 2001, Bautista-Cruz et al. 2014, Berch \& Kendrick 1982, Berliner \& Torrey 1989, Bidartondo et al., 2011, Carrillo, Godoy, \& Peredo, 1992, Castillo, Borie, Godoy, Rubio, \& Sieverding, 2006, Cooke \& Lefor 1998, Cooper 1976, de la Rosa-Mera et al., 2012, de Marins et al., 2009, Dhillion, 1993, Fernández, Messuti, \& Fontenla, 2013, Gemma et al., 1992, Hepden, 1960, Iqbal, Yousaf, \& Younus, 1981, Kessler et al., 2009, Kessler et al., 2014, Khade \& Rodrigues, 2002, Kovács, Kottke, \& Oberwinkler, 2003, Kovács, Balazs, \& Penzes, 2007, Laferrière \& Koske, 1981, Lara-Pérez et al., 2014, Lara-Pérez, 2007, Lara-Pérez, 2015, Laursen, Treu, Seppelt, \& Stephenson, 1997, Lee, Eom, Lee, \& Lee, 2001, Lehnert et al., 2009, Lesica \& Antibus, 1990, Leung, Ye, \& Wong, 2007, Liu, Zhu, Chen, Christie, \& Li, 2005, Martínez, Chiocchio, Em, Rodríguez, \& Godeas 2012, Mishra, Sharma, \& Gatphoh, 1980, Moteetee, Duckett, \& Russell, 1996, Muthukumar, Sathiyaraj, Priyadharsini, Uma, \& Sathiyadash, 2014, Muthukumar \& Prabha 2012, Muthukumar \& Prabha 2013, Muthuraja, Muthukumar, Sathiyadash, Uma, \& Priyadharsini, 2014, Nadarajah \& Nawawi, 1993, Palmieri \& Swatzell, 2004, Reyes-Jaramillo et al., 2008, Rimington et al., 2015, Santhoshkumar \& Nagarajan 2014, Schmid \& Oberwinkler, 1996, Schmid, Oberwinkler, \& Gómez, 1995, Schmid \& Oberwinkler, 1993, Schneider, Oliveira, Guilherme, Stürmer, \& Soares, 2012, Seerangan \& Thangavelu, 2014, Treu, Laursen, Stephenson, Landolt, \& Densmore, 1996, Trotta et al., 2006, Turnau et al., 2005, West, Brandt, Holstien, Hill, \& Hill, 2009, Winther \& Friedman, 2009, Winther \& Friedman 2008, Yi, Fu, Cao, \& Ding, 1994, Zhang, Guo, \& Liu, 2004, Zhao, 2000, Zubek et al., 2010.

muestras el $76 \%$ presentó colonización por hongos micorrízicos, el $52.56 \%$ se registró la presencia de arbúsculos, el $10.25 \%$ únicamente presentó colonización en forma de hifas, vesículas, o coils, el $23.93 \%$ estuvieron ausentes de colonización, el $20 \%$ fue por HSO, la mitad (9.82 \%) compartido con HMA y el $0.85 \%$ por Mucoromycotina (Cuadro 1, Fig. 1A).

De las 137 especies, el $68.61 \%$ corresponden a especies terrestres, el $23.35 \%$ a epífitas, $4.37 \%$ a saxícolas y el $3.64 \%$ a acuáticas. El $78.1 \%$ de las especies presentó colonización por hongos, el $56.2 \%$ fueron por micorriza arbuscular, $8.9 \%$ solo presentó colonización por hifas y/o vesículas, el $21.89 \%$ no presentaron colonización, mientras que el $29.9 \%$ fue por HSO y el $1.4 \%$ por Mucoromycotina (Fig. 1B). Comparando por el tipo de sustrato, el número de muestras que presentaron mayor colonización por micorriza arbuscular fueron las especies terrestres $64.5 \%$, saxícolas $50 \%$, acuáticas $20 \%$ y epífitas $5.41 \%$. La colonización por HSO se presentó en mayor porcentaje en las especies acuáticas $40 \%$, seguida de las epífitas $29.7 \%$, las terrestres $18 \%$ y las saxícolas $7.14 \%$ (Fig. 2A). En el número de especies registradas, la mayor colonización se observó en especies terrestres $76.59 \%$, saxícolas 33.33 $\%$, acuáticas $20 \%$ y epífitas $6.25 \%$. Mientras que la colonización por HSO fue mayor en especies acuáticas con $80 \%$, epífitas $37.5 \%$, terrestres $25.5 \%$ y saxícolas $16.6 \%$ (Fig. 2B).

Las especies con mayor número de muestras fueron Pteris vittata L. con 12 registros, Pteridium aquilinum (L.) Kuhn in v. d. Decken con nueve, Adiantum capillus-veneris L. con ocho, Botrychium virginianum (L.) Sw. con seis, Pteris cretica L. y Psilotum nudum (L.) 


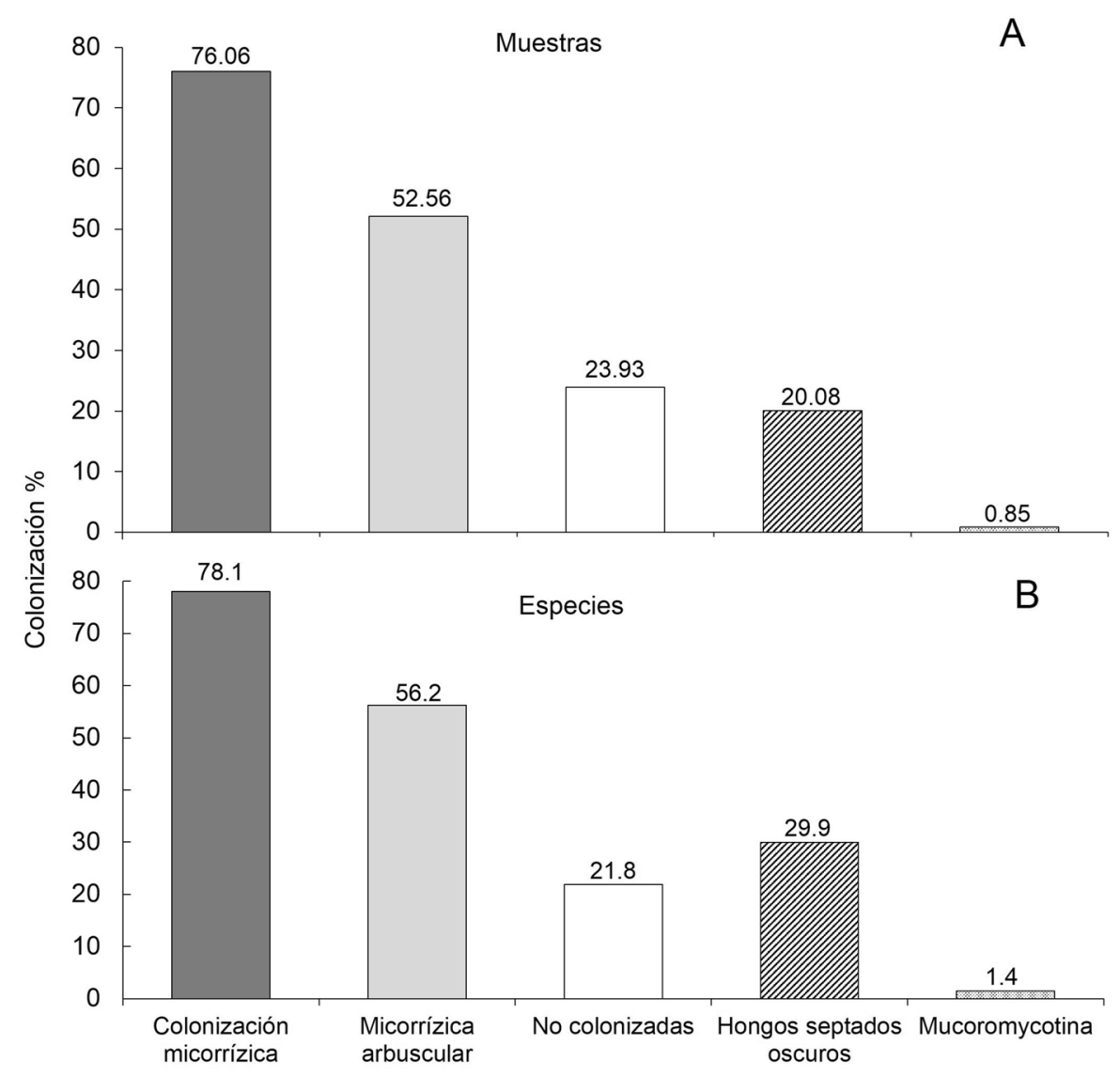

Fig. 1. Porcentaje de la colonización de la micorriza arbuscular, Mucoromycotina y hongos septados en de helechos y licófitas con distribución en México compilada de la revisión de literatura. A. Muestras. B. Especies.

Fig. 1. Percentage of arbuscular mycorrhizal, Mucoromyconina and dark septate fungi colonization ferns and lycophytes with distribution in Mexico compiled from literature review. A. Samples. B. Species.

P. Beauv. con cinco registros (Cuadro 1). El resto está representado con uno a cuatro registros (Cuadro 1). Las familias de helechos y licófitas con mayor número de especies fueron Pteridaceae con 28, que corresponde al $12 \%$ de las especies que contiene la familia en México, Polypodiaceae con 22 especies (17\%), seguida de Aspleniaceae con 13 (14\%) y Dryopteridaceae con 10 (7.1 \%) (Cuadro 2). Para las familias Dicksoniaceae, Metaxyaceae, Psilotaceae y Saccolomataceae se obtuvo información para el $100 \%$ de las especies con distribución en México, si bien cabe mencionar que estas familias solo están representadas por una o dos especies (Cuadro 2). Sin embargo, para las familias Cibotiaceae, Culcitaceae, Isoëtaceae, Lindsaeaceae, Lygodiaceae, Oleandraceae, Onocleaceae, Plagiogyriaceae y Tectariaceae no se encontró ningún registro sobre las interacciones endomicorrízicas (Cuadro 2).

El $61 \%$ de los trabajos incluidos en la revisión estuvieron dirigidos a estimar la colonización micorrízica en helechos y licófitas, seguido de estudios realizados con plantas vasculares en general (18.6 \%). La información restante está repartida en estudios de ecosistemas acuáticos, epífitas y en plantas que crecen en suelos contaminados con metales pesados. Los estudios se enfocaron principalmente a la fase esporofítica, ya que únicamente en ocho 


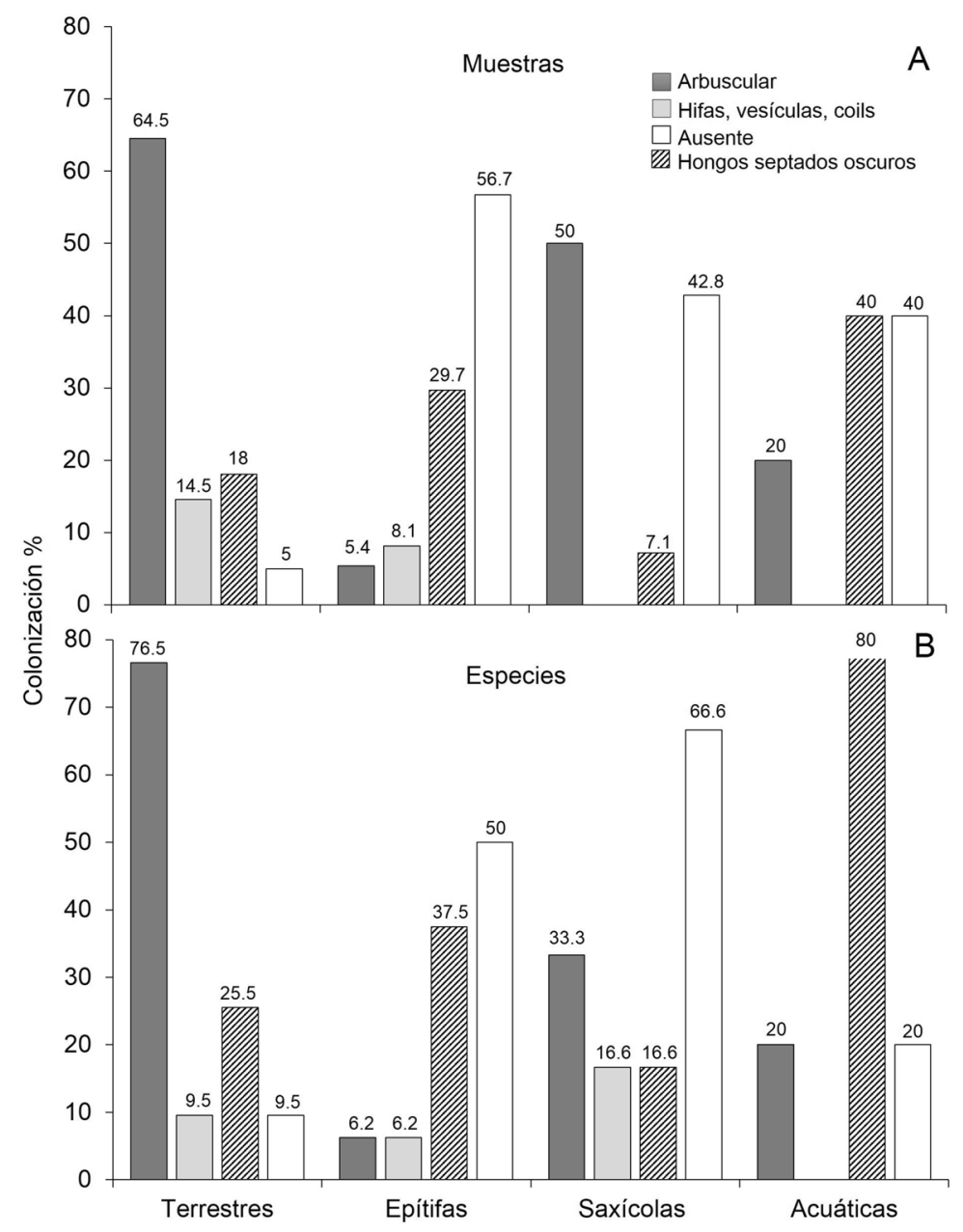

Fig. 2. Porcentaje de colonización por micorriza arbuscular y hongos septados oscuros en helechos y licófitas terrestres, acuáticas, saxícolas y acuáticas con distribución en México compilada de la revisión de literatura. A. Muestras. B. Especies. Fig. 2. Percentage arbuscular mycorrhizal and dark septate fungi colonization in terrestrial, epiphytic, saxicolous, and aquatic ferns and lycophytes with distribution in Mexico compiled from literature review. A. Samples. B. Species.

trabajos $(13.5 \%)$ se analizaron gametofitos. La metodología para evidenciar la colonización endomicorrízica en la mayoría de los trabajos examinados $(87.8 \%)$ fue mediante técnicas estándares de clareo y tinción de raíz y con apenas un $10 \%$ mediante la confirmación molecular.

\section{DISCUSIÓN}

La información aquí presentada sobre la colonización de hongos micorrízicos arbusculares, Mucoromycotina y hongos septados oscuros en helechos y licófitas correspondió al $77 \%$ de las familias, $91 \%$ de los géneros, y el $13.37 \%$ de las especies registradas para México (Mickel \& Smith, 2004). Los estudios sobre interacciones micorrízicas realizadas en México es de apenas el $2.7 \%$ de la riqueza de helechos y licófitas, y aporta el $20 \%$ de las especies registradas en este estudio (Reyes-Jaramillo, Camargo-Ricalde, \& Aquiahuatl-Ramos, 2008; de la Rosa-Mera, Ferrera-Cerrato, Alarcón, Sánchez-Colín, 
CUADRO 2

Colonización de hongos en las familias de helechos y licófitas con distribución en México

TABLE 2

Fungal colonization among families of ferns and lycophytes with distribution in Mexico

\begin{tabular}{|c|c|c|c|}
\hline Familia & $\begin{array}{l}\text { Especies en } \\
\text { México }\end{array}$ & $\begin{array}{c}\text { Especies } \\
\text { analizadas }\end{array}$ & Porcentaje \\
\hline Anemiaceae & 20 & 2 & 10 \\
\hline Aspleniaceae & 89 & 13 & 14.6 \\
\hline Blechnaceae & 19 & 5 & 26.3 \\
\hline Cibotiaceae & 2 & - & - \\
\hline Culcitaceae & 1 & - & - \\
\hline Cyatheaceae & 14 & 4 & 28.6 \\
\hline Dennstaedtiaceae & 22 & 4 & 18.2 \\
\hline Dicksoniaceae & 2 & 2 & 100 \\
\hline Dryopteridaceae & 140 & 10 & 7.1 \\
\hline Equisetaceae & 3 & 2 & 66.7 \\
\hline Gleicheniaceae & 7 & 4 & 57.1 \\
\hline Hymenophyllaceae & 48 & 7 & 14.6 \\
\hline Isoetaceae & 6 & - & - \\
\hline Lindsaeaceae & 9 & - & - \\
\hline Lomariopsidaceae & 12 & 3 & 25 \\
\hline Lycopodiaceae & 20 & 5 & 25 \\
\hline Lygodiaceae & 3 & - & - \\
\hline Marattiaceae & 6 & 1 & 16.7 \\
\hline Marsileaceae & 8 & 2 & 25 \\
\hline Metaxyaceae & 1 & 1 & 100 \\
\hline Oleandraceae & 1 & - & - \\
\hline Onocleaceae & 1 & - & - \\
\hline Ophioglossaceae & 14 & 3 & 21.4 \\
\hline Osmundaceae & 2 & 1 & 50 \\
\hline Plagiogyriaceae & 1 & - & - \\
\hline Polypodiaceae & 129 & 22 & 17.1 \\
\hline Psilotaceae & 2 & 2 & 100 \\
\hline Pteridaceae & 218 & 28 & 12.8 \\
\hline Saccolomataceae & 2 & 2 & 100 \\
\hline Salviniaceae & 4 & 3 & 75 \\
\hline Schizaeaceae & 3 & 1 & 33.3 \\
\hline Selaginellaceae & 80 & 3 & 3.8 \\
\hline Tectariaceae & 7 & - & - \\
\hline Thelypteridaceae & 70 & 4 & 5.7 \\
\hline Woodsiaceae & 42 & 3 & 7.1 \\
\hline Total & 1008 & 137 & \\
\hline
\end{tabular}

\& Franco-Ramírez, 2012; Bautista-Cruz, Montaño, Camargo-Ricalde, \& Pacheco, 2014; Lara-Pérez et al., 2015). La riqueza de especies de helechos y licófitas de las que se carece de información respecto a las interacciones micorrízicas, es cercana a las 900 especies. A nivel mundial, la proporción de especies de helechos y licófitas, para las que falta evaluar su estatus micorrízico, está entre el 89-90 \% (Kessler et al., 2009; Lehnert, Krug, \& Kessler, 2016), por lo que es necesario ampliar el número de especies analizadas para tener un conocimiento más completo de sus interacciones micorrízicas.

En el presente estudio, los hongos que colonizan a helechos y licófitas son los HMA, HSO y Mucoromycotina. En total, los tres grupos de hongos colonizan el $78.1 \%$ de las especies, no obstante, sólo el $56.2 \%$ pueden ser consideradas especies micorrízicas arbusculares debido a la presencia de arbúsculos, interface especializada para el intercambio bidireccional de nutrimentos (van der Heijden et al., 2015). El porcentaje de especies micorrízicas obtenido en este trabajo es similar a estimaciones realizadas por Wang y Qiu (2006) y Lehnert et al. (2009), quienes reportan que el 52 y $53 \%$ de las especies presentan colonización micorrízica arbuscular, respectivamente. Una reciente revisión mundial de las asociaciones micorrízicas en helechos y licófitas estima que la colonización por HMA en helechos y licófitas es del $54 \%$ (Lehnert et al., 2016). En este trabajo la mayoría de los datos de colonización micorrízica vienen de una sola muestra y no es necesariamente representativa del género o de la familia de helechos o licófitas. Por lo cual, es necesario analizar más individuos de las especies, sobre todo aquellas que presentaron colonización únicamente por hifas y vesículas para poder confirmar sus estatus micorrízico (Brundrett, 2009).

La proporción de especies micorrízicas arbusculares en helechos y licófitas es mayor en las especies terrestres $(76.5 \%)$ en comparación con las saxícolas (33.3\%), acuáticas $(20 \%)$ y epífitas $(6.2 \%)$. El bajo número de especies con colonización de micorriza arbuscular en especies saxícolas, acuáticas y epífitas, posiblemente se debe a la baja riqueza y abundancia de esporas de HMA, registrada en estos sustratos (Janos \& Sahley, 1995; McIlveen \& Cole, 1976; 
de Marins, Carrenho, \& Thomaz, 2009; Seerangan \& Thangavelu, 2014).

De manera general, la presencia de HSO se presenta en el $30 \%$ de helechos y licófitas; sin embargo, esta proporción sin duda variará dependiendo de los sustratos analizados (Andrade, Queiroz, Hermes, \& Oliveira, 2000; Lenhert et al., 2009; Zubek et al., 2010; Kessler, Güdel, Salazar, Homeier, \& Kluge, 2014). La presencia de HSO resultó ser mayor en helechos acuáticos (80\%) comparado con los epífitos $(37.5 \%)$, terrestres $(25.5 \%)$ y saxícolas (16.6\%). La proporción de especies colonizadas por HSO en vegetación acuática y subacuática es parecida al $79.16 \%$ reportado por de Marins et al. (2009); y contrasta con Seerangan y Thangavelu (2014) quienes reportan únicamente el $8.6 \%$ de colonización por HSO.

Algunos autores como Boullard (1979), Harley y Harley (1987), Gemma, Koske y Flynn (1992) y Muthukumar y Udaiyan (2000) han reportado la ausencia de colonización micorrízica arbuscular en helechos y licófitas acuáticas, sin embargo, Bajwa, Yaqoob y Javaid (2001) reportaron colonización micorrízica arbuscular en Marsilea vestita Hook. \& Grev., que presenta variación estacional con altos porcentajes de arbúsculos en otoño. Además, se ha constatado la presencia de hifas y vesículas de hongos micorrízicos en Marsilea quadrifolia L. y Salvinia natans (L.) All. (Bagyaraj, Manjunath, \& Patil, 1979; Radhika \& Rodrigues, 2007).

En México, hay 12 especies de helechos acuáticos o subacuáticos de las familias Marsileaceae (ocho especies) y Salvinaceae (cuatro especies), y alta diversidad especies vegetales en manglares, popales, tulares, carrizales y especies de la vegetación flotante y sumergida (Rzedowski, 1978; Montaño et al., 2012). Sin embargo, han sido pocos los estudios ecológicos para entender la riqueza y la dinámica de los HMA y los HSO en estas comunidades vegetales.

Las familias con mayor número de especies con información de las interacciones micorrízicas fueron Pteridaceae, Polypodiaceae, Dryopteridaceae y Aspleniaceae. Este resultado es esperado, ya que las mencionadas familias aportan más del $56 \%$ de la riqueza de helechos y licófitas de México (Mickel \& Smith, 2004). Por el contrario, es posible que las familias menos representadas en estudios de interacciones micorrízicas (Cibotiaceae, Culcitaceae, Isoëtaceae, Lindsaeaceae, Lygodiaceae, Oleandraceae, Onocleaceae, Plagiogyriaceae y Tectariaceae) sea debido a la baja riqueza de especies tanto a nivel mundial como en México (Mickel \& Smith, 2004; Smith et al., 2006).

En México hay 186 especies de helechos y licófitas endémicas y muchas especies más que se encuentran amenazadas, debido a la reducción de su hábitat tal y como ocurre con el bosque mesófilo de montaña (Mickel y Smith, 2004). En consecuencia, conocer el estatus micorrízico podría ser una estrategia alternativa para su propagación y conservación. Los reportes bibliográficos sobre las interacciones micorrízicas en helechos y licófitas emplearon la fase esporofítica ( $80 \%$ ), fase conspicua con caracteres taxonómicos de relevancia a diferencia de la gametofítica. La determinación de la presencia de hongos en la fase gametofítica es necesaria para entender el papel que juegan los diferentes grupos taxonómicos de hongos en el establecimiento de helechos y licófitas en condiciones naturales, y conocer si existe una fluctuación en la colonización micorrízica en los hábitats con marcada estacionalidad.

El gametofito es una etapa crítica y vulnerable a la depredación, desecación, y pocas oportunidades de luz en sotobosque, por lo que la micorriza puede ser una estrategia para el reclutamiento de las especies en los diferentes ecosistemas (Leake, 1994). La dependencia obligada de la micorriza arbuscular en gametofitos aclorófilos subterráneos micoheterotróficos es evidente (Winther \& Friedman, 2008, 2009; Field et al. 2015). En cambio, los gametofitos cordados fotosintéticos se han considerado ausentes de colonización (Campbell, 1908; Boullard, 1979; Schmid \& Oberwinkler, 1993). Para un tener un panorama completo de las interacciones gametofito-hongo en el ciclo de vida de los helechos y licófitas, hay que tener en cuenta los tipos de ambientes donde 
se desarrollan las especies. Estudios recientes, confirman la presencia de micorriza arbuscular en gametofitos cordados de helechos, que crecen al interior de un bosque subtropical perennifolio en Japón (Ogura-Tsujita et al., 2015). Por el contrario, gametofitos de las especies Pteris vittata y Nephrolepis exaltata, consideradas como especies pioneras, demuestran que no son susceptibles a la colonización por HMA, formando micorriza únicamente en el esporofito diploide (Lloyd, 1974; Bonfante \& Selosse, 2010; Wang et al., 2012; Muthukumar \& Prabha 2012). Las herramientas moleculares pueden ayudarnos en la identificación taxonómica de las especies de hongos que colonizan tanto a gametofitos como a helechos y licófitas (Pryer et al., 2004; Ogura-Tsujita et al., 2015). Para tal fin, una alternativa sería implementar estudios experimentales obteniendo gametofitos a partir de la siembra de esporas (Turnau et al., 2005; Lara-Pérez, 2007; Reyes-Jaramillo et al., 2008).

El estudio de los hongos Mucoromycotina está iniciando y por ahora se encuentra representado en las familias Lycopodiaceae y Ophioglosaceae pero, conforme avancen los estudios en este grupo de hongos, seguramente se revelarán nuevos registros en muchas otras familias de helechos y licófitas (Rimington et al., 2015). Debido a que la morfología de los Mucoromycotina es muy parecida a los Glomeromycota, sería necesario incorporar metodologías moleculares, que nos permitan diferenciar las especies de los grupos taxonómicos asociados para evitar registros erróneos (Bidartondo et al., 2011; Rimington et al., 2015). Los HSO como los Mucoromycotina podrían tener amplia relevancia ecológica, sin embargo, es necesario establecer estudios experimentales para evidenciar su funcionalidad.

Finalmente, se considera imprescindible realizar estudios comparativos de la interacción micorrízica en gametofitos y esporofitos de helechos y licófitas en los diferentes ambientes y sustratos donde se desarrollan, para entender la relevancia ecológica de los HMA en la adecuación de las especies. Para tener un mejor entendimiento de las interacciones de los hongos que colonizan a los helechos y licófitas se requiere de estudios complementarios que incluyan estudios de biología molecular, fisiología y ecología. Dentro de los países del trópico, México se distingue por tener una alta riqueza de helechos y licófitas con especies endémicas, aunadas a un mosaico ambiental muy heterogéneo, por lo que es un escenario ideal para llevar a cabo investigaciones ecológicas de los diferentes hongos que colonizan a helechos y licófitas.

\section{AGRADECIMIENTOS}

El primer autor agradece al CONACYT por la beca (No. 223899) para estudios Doctorales en el Instituto de Biotecnología y Ecología Aplicada, durante la cual se realizó la primera etapa de este trabajo. Los autores agradecen a los tres revisores anónimos por sus valiosas aportaciones y sugerencias a la versión inicial del manuscrito.

\section{RESUMEN}

Los helechos y licófitas son un grupo de plantas vasculares de interés para entender la evolución de las interacciones micorrízicas y su preservación es de relevancia por sus múltiples interacciones ecológicas. El registro de los diferentes grupos taxonómicos de hongos asociados a helechos y licófitas se encuentra fragmentado y el criterio para su diagnóstico inconsistente, lo que dificulta el entendimiento y la determinación del estatus micorrízico. El objetivo del presente trabajo fue conocer el porcentaje de especies de helechos y licófitas que tiene distribución en México de las que se tiene información sobre hongos endomicorrízicos. Se integró un listado de la presencia de hongos micorrízicos arbusculares (HMA), Mucoromycotina y hongos septados oscuros (HSO) asociados a helechos y licófitas, a través de una búsqueda exhaustiva de información. En este trabajo se consideró una especie micorrízica arbuscular, por los arbúsculos, para diferenciarla de la colonización por hifas, vesículas u ovillos. El estudio generó un listado de ocurrencia de interacciones endomicorrízicas en 27 familias, 61 géneros y 137 especies de helechos y licófitas, que representa el $13.37 \%$ de las especies, $91 \%$ de los géneros y $77 \%$ de las familias registradas para México. El $78.1 \%$ de las especies presentó colonización por hongos, el $56.2 \%$ fueron por HMA, el $8.9 \%$ solo presentó colonización por hifas, vesículas o coils, el $21.8 \%$ fue por HSO y el 1. $4 \%$ por hongos Mucoromycotina. Del total de especies, la mayor presencia de colonización se presentó en especies 
terrestres $76.59 \%$, saxícolas $33.33 \%$, acuáticas $20 \%$ y epífitas con $6.25 \%$. Las familias de helechos y licófitas con mayor número de especies colonizadas fueron: Pteridaceae, Polypodiaceae, Aspleniaceae y Dryopteridaceae. El presente trabajo muestra que la asociación de HMA y HSO es la más ampliamente distribuida en las familias y especies de helechos y licófitas. Surge la necesidad de incluir a los helechos y licófitas en los estudios de interacciones endomicorrízicas ya que únicamente 28 especies (20\%) fueron estudiadas en ecosistemas de México. La mayoría de los estudios se enfocan en la fase esporofítica (80 \%). Sin embargo, para entender el papel que juegan las micorrizas en el establecimiento de helechos y licófitas se requiere incluir a la fase gametofítica, así como estudios de ecología, biología molecular, así como trabajos experimentales de fisiología. La información presentada es de relevancia para implementar estrategias alternativas de conservación, debido a que muchas especies de helechos y licófitas requieren de la asociación micorrízica para su crecimiento y supervivencia.

Palabras clave: micorriza arbuscular, hongos septados oscuros, Murocomycota, Licófitas, Monilofitas, helechos.

\section{REFERENCIAS}

Alarcón, A., Hernández-Cuevas, L. V., Ferrera-Cerrato, R., \& Franco-Ramírez, A. (2012). Diversity and agricultural applications of arbuscular mycorrhizal fungi in Mexico. Journal of Fertilizers Pesticides, 3, 115 doi:104172/2155-62021000115

Andrade, A. C. S., Queiroz, M. H., Hermes, R. A. L., \& Oliveira, V. L. (2000). Mycorrhizal status of some plants of the araucaria forest and the Atlantic rainforest in Santa Catarina, Brazil. Mycorrhiza, 10, 131-136.

Bagyaraj, D. J., Manjunath, A., \& Patil, R. B. (1979). Occurrence of vesicular-arbuscular mycorrhizas in some tropical aquatic plants. Transactions of the British Mycological Society, 72, 164-167.

Bajwa, R., Yaqoob, A., \& Javaid, A. (2001). Seasonal variation in VAM in wetland plants Pakisan. Journal of Biological Sciences, 4, 464-470.

Bautista-Cruz, A. A., Montaño, N. M., Camargo-Ricalde, S. L, \& Pacheco, L. (2014). Hongos micorrizógenos arbusculares y nutrimentos del suelo asociados a cuatro especies de helechos en dos ecosistemas de Oaxaca, México. Revista Chapingo. Serie ciencias forestales y del ambiente, 20, 199-212.

Berch, S. M., \& Kendrick, B. (1982). Vesicular-arbuscular mycorrhizae of southern Ontario ferns and ferns allies. Mycologia, 74, 769-776.
Berliner, R., \& Torrey, J. G. (1989). Studies on mycorrhizal associations in Harvard Forest, Massachusetts. Canadian Journal of Botany, 67, 2245-2251.

Bidartondo, M. I., Read, D. J., Trappe, J. M., Merckx, V., Ligrone, R., \& Duckett, J. G. (2011). The dawn of symbiosis between plants and fungi. Biology Letters, 7, 574-577.

Bonfante, P., \& Genre, A. (2008). Plants and arbuscular mycorrhizal fungi: an evolutionary-developmental perspective. Trends in Plant Science, 13, 492-498.

Bonfante, P., \& Selosse, M. A. (2010). A glimpse into the past of land plants and of their mycorrhizal affairs: from fossils to evo-devo. New Phytologist, 186, 267-270.

Boullard, B. (1979). Considérations sur les symbioses fongiques chez les Ptéridophytes. Syllogeus, 19, 1-58.

Brundrett, M. C. (2002). Coevolution of roots and mycorrhizas of land plants. New Phytologist, 154, 275-304.

Brundrett, M. C. (2009). Mycorrhizal associations and other means of nutrition of vascular plants: understanding the global diversity of host plants by resolving conflicting information and developing reliable means of diagnosis. Plant and Soil, 320,37-77.

Campbell, D. H. (1908). Symbiosis in fern protallia. The American Naturalist, 42, 154-165.

Carrillo, R., Godoy, R., \& Peredo, H. (1992). Simbiosis micorrícica en comunidades boscosas del Valle Central en el sur de Chile. Bosque, 13, 57-67.

Castillo, C. G., Borie, F., Godoy, R., Rubio, R., \& Sieverding, E. (2006). Diversity of mycorrhizal plant species and arbuscular mycorrhizal fungi in evergreen forest, deciduous forest and grassland ecosystems of Southern Chile. Journal of Applied Botany and Food Quality, 80, 40-47.

Christenhusz, M. J. M., Zhang, X. C., \& Schneider, H. (2011). A linear sequence of extant families and genera of lycophytes and ferns. Phytotaxa, 19, 7-54.

Cooke, J. C., \& Lefor, M. W. (1998). The mycorrhizal status of selected plant species from Connecticut wetlands and transition zones. Restoration Ecology, $6,214-222$

Cooper, K. M. (1976). A field survey of mycorrhizas in New Zealand ferns. New Zealand Journal of Botany, 14, 169-181.

de la Rosa-Mera, C., Ferrera-Cerrato, R., Alarcón, A., Sánchez-Colín, M. J., \& Franco-Ramírez, A. (2012). Aislamiento de consorcios de hongos micorrícicos arbusculares de plantas medicinales y su efecto en el crecimiento de vinca (Catharanthus roseus). Revista Chilena de Historia Natural, 85, 187-198. 
de Marins, J. F., Carrenho, R., \& Thomaz, S. M. (2009). Occurrence and coexistence of arbuscular mycorrhizal fungi and dark septate fungi in aquatic macrophytes in a tropical river-floodplain system. Aquatic Botany, 91, 13-19.

Dhillion, S. S. (1993). Vesicular-arbuscular mycorrhizas of Equisetumspecies in Norway and the USA -occurrence and mycotrophy. Mycological Research, 97, 656-660.

Fernández, N., Messuti, M. I., \& Fontenla, S. (2008). Arbuscular mycorrhizas and dark septate fungi in Lycopodium paniculatum (Lycopodiaceae) and Equisetum bogotense (Equisetaceae) in a Valdivian temperate forest of Patagonia, Argentina. American Fern Journal, 98, 117-127.

Fernández, N. V., Messuti, M. I., \& Fontenla, S. B. (2013). Occurrence of arbuscular mycorrhizas and dark septate endophytes in pteridophytes from a patagonian rainforest, Argentina. Journal of Basic Microbiology, $53,498-508$.

Field, K. J., Leake, Jr., Tille, S., Allinson, K. E., Rimington, W. R., Bidartondo, M. I., Beerling, D. J., \& Cameron, D. D. (2015). From mycohetereotrophy to mutualism: mycorrhizal specificity and functioning in Ophioglossum vulgatum sporophytes. New Phytologist, 205, 1492-1502.

Gemma, J. N., Koske, R. E., \& Flynn, T. (1992). Mycorrhizae in Hawaiian pteridophytes: occurrence and evolutionary significance. American Journal of Botany, $79,843-852$.

Harley, J. L., \& Harley, E. L. (1987). A check-list of mycorrhiza in the British flora. New Phytologist Supplement, 105, 1-100.

Hepden, P. M. (1960). Studies in vesicular-arbuscular endophytes II Endophytes in the Pteridophyta, with special references to leptosporangiate ferns. Transactions of the British Mycological Society, 43, 559-570.

Iqbal, S. H, Yousaf, M., \& Younus, M. (1981). A field survey of mycorrhizal associations in ferns of Pakistan. New Phytologist, 87, 69-79.

James, T. Y., Kauff, F., Schoch, C. L., Matheny, P. B., Hofstetter, V., Cox, ... \& Vilgalys, R. (2006). Reconstructing the early evolution of fungi using a six-gene phylogeny. Nature, 443, 818-822.

Janos, D. P., \& Sahley, C. T. (1995). Rodent dispersal of vesicular-arbuscular mycorrhizal fungi in amazonian Peru. Ecology, 76, 1852-1858.

Jumpponen, A. (2001). Dark septate endophyte -are they mycorrhizal. Mycorrhiza, 11, 207-211.

Kessler, M., Güdel, R., Salazar, L., Homeier, J., \& Kluge, J. (2014). Impact of mycorrhization on the abundance, growth and leaf nutrient status of ferns along a tropical elevational gradient. Oecologia, 175, 887-900.

Kessler, M., Jonas, R., Cicuzza, D., Kluge, J., Piatek, K., Naks, P., \& Lehnert, M. (2009). A survey of the mycorrhization of Southeast Asian ferns and lycophytes. Plant Biology, 12, 788-793.

Kessler, M., Jonas, R., Strasberg, D., \& Lehnert, M. (2010). Mycorrhizal colonizations of ferns and lycophytes on the island of La Réunion in relation to nutrient availability. Basic and Applied Ecology, 11, 329-336.

Khade, S. W., \& Rodrigues, B. F. (2002). Arbuscular mycorrhizal fungi associated with some pteridophytes from western ghat region of Goa. Tropical Ecology, $43,251-256$.

Kottke, I., Haug, I., Setaro, S., Suárez, J. P., Weiß, M., Preußing, M., Nebel, M., \& Oberwinkler, F. (2008). Guilds of mycorrhizal fungi and their relation to trees, ericads, orchids and liverworts in a neotropical mountain rain forest. Basic and Applied Ecology, 9, 13-23.

Kovács, G. M., Balazs, T., \& Penzes, Z. (2007). Molecular study of arbuscular mycorrhizal fungi colonizing the sporophyte of the eusporangiate rattlesnake fern (Botrychium virginianum, Ophioglossaceae). Mycorrhiza, 17, 597-605.

Kovács, G. M., Kottke, I., \& Oberwinkler, F. (2003). Light and electron microscopic study on the mycorrhizae of sporophytes of Botrychium virginianum-arbuscular structure resembling fossil forms. Plant Biology, $5,574-580$

Laferrière, J. L., \& Koske, R. E. (1981). Occurrence of VA-mycorrhizas in some Rhode Island Pteridophytes. Transactions of the British Mycological Society, 76, 331-332.

Lara-Pérez, L. A. (2007). Efecto de los hongos micorrizógenos arbusculares en la supervivencia de gametofitos micropropagados de Cyathea fulva Pteridophyta: Cyatheaceae (Tesis de Licenciatura). Universidad Veracruzana, México.

Lara-Pérez, L. A., Noa-Carrazana, J. C., López-Landa, J., Hernández-González, S., Oros-Ortega I., \& AndradeTorres, A. (2014). Colonization and structure of arbuscular mycorrhizal fungi community in Alsophila firma (Cyatheales: Cyatheaceae) from a tropical montane cloud forest in Veracruz, México. Revista de Biología Tropical, 62, 1609-1623.

Lara-Pérez, L. A., Valdés-Baizabal, M. D., Noa-Carrazana, J. C., Zulueta-Rodríguez, R., Lara-Capistrán, L., \& Andrade-Torres, A. (2015). Mycorrhizal associations of ferns and lycopods of central Veracruz, Mexico. Symbiosis, 65, 185-192. 
Laursen, G. A., Treu, R., Seppelt, R. D., \& Stephenson, S. L. (1997). Mycorrhizal assessment of vascular plants from subantarctic Macquarie Island. Arctic and Alpine Research, 29, 483-491.

Leake, J. R. (1994). The biology of mycoheterotrophic ('saprophitic') plants. New Phytologist, 127, 171-216.

Lee, J. K., Eom, A. H., Lee, S. S, \& Lee, C. H. (2001). Mycorrhizal symbioses found in roots of fern and its relatives in Korea. Journal of Plant Biology, 44, $81-86$.

Lehnert, M., Kottke, I., Setaro, S., Pazmino, L. F., Suárez, J. P., \& Kessler M. (2009). Mycorrhizal associations in ferns from Southern Ecuador. American Fern Journal, 99, 292-306.

Lehnert, M., Krug, M., \& Kessler, M. (2016). A review of symbiotic fungal endophytes in lycophytes and ferns-a global phylogenetic and ecological perspective. Symbiosis, 1-13. doi:10.1007/s13199-016-0436-5

Lesica, P., \& Antibus, R. K. (1990). The occurrence of mycorrhizae in vascular epiphytes of two Costa Rican rain forests. Biotropica, 33, 250-258.

Leung, H. M., Ye, Z. H., \& Wong, M. H. (2007). Survival strategies of plants associated with arbuscular mycorrhizal fungi on toxic mine tailings. Chemosphere, 66, 905-915.

Liu, Y., Zhu, Y. G., Chen, B. D., Christie, P., \& Li, X. L. (2005). Influence of the arbuscular mycorrhizal fungus Glomus mosseae on uptake of arsenate by the As hyperaccumulator fern Pteris vittata L. Mycorrhiza, 15, 187-192.

Lloyd, R. M. (1974). Mating systems and genetic load in pioneer and non-pioneer Hawaiian Pteridophyta. Botanical Journal of the Linnean Society, 69, 23-35.

Martínez, A. E., Chiocchio, V., Em, L. T., Rodríguez, M. A., \& Godeas, A. M. (2012). Mycorrhizal association in gametophytes and sporophytes of fern Pteris vittata (Peridaceae) with Glomus intraradices. Revista de Biología Tropical, 60, 857-865.

McIlveen, W. D., \& Cole, Jr., H. (1976). Spore dispersal of Endogonaceae by worms, ants, wasps, and birds. Canadian Journal of Botany, 54, 1486-1489.

Mehltreter, K. (2010). Interactions of ferns with fungi and animals. In K. Mehltreter, L. R. Walker, \& J. M. Sharpe (Eds.), Fern Ecology (pp. 220-254). Cambridge: Cambridge University Press.

Merckx, V., \& Bidartondo, M. I. (2008). Breakdown and delayed cospeciation in the arbuscular mycorrhizal mutualism. Proceedings of the Royal Society B: Biological Sciences, 275, 1029-1035.
Mickel, J. T., \& Smith, A. R. (2004). The pteridophytes of Mexico. Memoirs of the New York Botanical Garden, $88,1-1054$

Mishra, R. R., Sharma, G. D., \& Gatphoh, A. R. (1980). Mycorrhizas in the ferns of North Eastern India. Proceedings of the Indian Academy of Science, 46, 546-551.

Montaño, N. M., Alarcón, A., Camargo-Ricalde, S. L., Hernández-Cuevas, L. V., Álvarez-Sánchez, J., González-Chávez, M. D. C. A., ... \& Ferrera-Cerrato, R. (2012). Research on arbuscular mycorrhizae in Mexico: an historical synthesis and future prospects. Symbiosis, 57, 111-126.

Moran, R. C. (2008). Diversity, biogeography and floristics. In: T. A. Ranker, \& C. H. Haufler, (Eds.), Biology and evolution of ferns and lycophytes (pp. 367-394). Cambridge, UK: Cambridge University Press.

Moteetee, A., Duckett. J. G., \& Russell, A. J. (1996). Mycorrhizas in the ferns of Lesotho. In J. M. Camus, M. Gibby, \& R. J. Johns (Eds.), Pteridology in perspective (pp. 621-632). UK: Royal Botanic Gardens, Kew.

Muthukumar, T., \& Prabha, K. (2012). Fungal associations in gametophytes and young sporophytic roots of the fern Nephrolepis exaltata. Acta Botanica Croatica, 71, 139-146.

Muthukumar, T., \& Prabha, K. (2013). Arbuscular mycorrhizal and septate endophyte fungal associations in lycophytes and ferns of South India. Symbiosis, $59,15-33$

Muthukumar, T., Sathiyaraj, G., Priyadharsini, P., Uma, E., \& Sathiyadash, K. (2014). Arbuscular mycorrhizal and dark septate endophyte fungal associations in ferns and lycophytes of Palni Hills, Western Ghats, southern India. Brazilian Journal of Botany, $37,561-581$.

Muthukumar, T., \& Udaiyan, K. (2000). Arbuscular mycorrhizas of plants growing in the Western Ghats region, southern India. Mycorrhiza, 9, 297-313.

Muthuraja, R., Muthukumar, T., Sathiyadash, K., Uma, E., \& Priyadharsini, P. (2014). Arbuscular mycorrhizal (AM) and dark septate endophyte (DSE) fungal association in lycophytes and ferns of the Kolli Hills Eastern Ghats, Southern India. American Fern Journal, 104, 67-102.

Nadarajah, P., \& Nawawi, A. (1993). Mycorrhizal status of epiphytes in Malaysian oil palm plantations. Mycorrhiza, 4, 21-25.

Newman, E. I., \& Reddell, P. (1987). The distribution of mycorhizas among families of vascular plants. New Phytologist, 106, 745-751. 
Newsham, K. K. (2011). A meta-analysis of plant responses to dark septate root endophytes. New Phytologist, 190, 783-793.

Ogura-Tsujita, Y., Hirayama, Y., Sakoda, A., Suzuki, A., Ebihara, A., Morita, N., \& Imaichi, R. (2015). Arbuscular mycorrhizal colonization in field-collected terrestrial cordate gametophytes of pre-polypod leptosporangiate ferns (Osmundaceae, Gleicheniaceae, Plagiogyriaceae, Cyatheaceae). Mycorrhiza, $26,87-97$.

Palmieri, M., \& Swatzell, L. J. (2004). Mycorrhizal fungi associated with the fern Cheilanthes lanosa in southeast Missouri and southern Illinois. Northeastern Naturalist, 11, 57-66.

Pirozynski, K. A., \& Malloch, D. W. (1975). The origin of land plants: a matter of mycotrophism. Biosystems, $6,153-164$.

Pryer, K. M., Schuettpelz, E., Wolf, P. G., Schneider, H., Smith, A. R., \& Cranfill, R. (2004). Phylogeny and evolution of ferns monilophytes with a focus on the early leptosporangiate divergences. American Journal of Botany, 91, 1582-1598.

Radhika, K. P. \& Rodrigues, B. F. (2007). Arbuscular mycorrhizae in association with aquatic and marshy plant species in Goa, India. Aquatic Botany, 86, 291-294

Raghavan, V. (1992). Germination of fern spores. American Scientist, 80, 176-185.

Redecker, D., Kodner, R., \& Graham, L. E. (2000). Glomalean fungi from the Ordovician. Science, 289, 1920-1921.

Reyes-Jaramillo, I., Camargo-Ricalde, S. L., \& AquiahuatlRamos, M. A. (2008). Mycorrhizal-like interaction between gametophytes and young sporophytes of the fern Dryopteris muenchii (Filicales) and its fungal endophyte. Revista de Biología Tropical, 56, 1101-1107.

Rimington, W. R., Pressel, S., Duckett, J. G., \& Bidartondo, M. I. (2015). Fungal associations of basal vascular plants: reopening a closed book? New Phytologist, $205,1394-1398$

Rzedowski, J. (1978). La Vegetación de México. Ciudad de México: Limusa.

Santhoshkumar, S., \& Nagarajan, N. (2014). AM fungal association in the rhizosphere soil of some pteridophytic plant species in Valparai Hills, Western Ghats of Tamilnadu, India. International Journal of Life Sciences, 2, 201-206.

Schmid, E., \& Oberwinkler, F. (1993). Mycorrhiza-like interaction between the achlorophyllous gametophyte of Lycopodium clavatum L. and its fungal endophyte studied by light and electron microscopy. New Phytologist, 124, 69-81.

Schmid, E., \& Oberwinkler, F. (1996). Light and electron microscopy of a distinctive VA mycorrhiza in mature sporophytes of Ophioglossum reticulatum. Mycological Research, 100, 843-849.

Schmid, E., Oberwinkler, F., \& Gómez, L. D. (1995). Light and electron microscopy of a host-fungus interaction in the roots of some epiphytic ferns from Costa Rica. Canadian Journal of Botany, 73, 991-996.

Schneider, J., Oliveira, L. M., Guilherme, L. R. G., Stürmer, S. L., \& Soares, C. R. F. S. (2012). Espécies tropicais de pteridófitas em associação com fungos micorrízicos arbusculares em solo contaminado com arsênio. Química Nova, 35, 709-714.

Schüßler, A., \& Walker, C. (2010). The Glomeromycota: a species list with new families and new genera Gloucester. England: The Royal Botanic Garden Edinburgh/The Royal Botanic Garden Kew/Botanische Staatssammlung Munich/Oregon State University.

Seerangan, K., \& Thangavelu, M. (2014). Arbuscular mycorrhizal and dark septate endophyte fungal associations in South Indian aquatic and wetland macrophytes. Journal of Botany, 1-14 doiorg/101155/2014/173125

Smith, A. R., Pryer, K. M., Schuettpelz, E., Korall, P., Schneider. H., \& Wolf, P. G. (2006). A classification for extant ferns. Taxon, 55, 705-731.

Smith, S. E., \& Read, D. J. (2008). Mycorrhizal Symbiosis New York: Academic Press.

Strullu-Derrien, C., Kenrick, P., Pressel, S., Duckett, J. G., Rioult, J. P., \& Strullu, D. G. (2014). Fungal associations in Horneophyton ligneri from the Rhynie Chert c 407 million year old closely resemble those in extant lower land plants: novel insights into ancestral plant-fungus symbioses. New Phytologist, 203, 964-979.

Tejero-Díez, J. D., Torres-Díaz, A., Mickel, J., Mehltreter, K., \& Krömer, T. (2009). Helechos y licófitas. In G. Ceballos, R. List, G. Garduño, R. López-Cano, M. J. Muñozcano-Quintanar, E. Collado, \& J. E. San Román (Eds.), La diversidad biológica del Estado de México: Estudio de Estado México (pp. 167176). México: Gobierno del Estado de México y Comisión Nacional para el Conocimiento y Uso de la Biodiversidad.

Treu, R., Laursen, G. A., Stephenson, S. L., Landolt, J. C., \& Densmore, R. (1996). Mycorrhizae from Denali National Park and Preserve, Alaska. Mycorrhiza, 6, 21-29.

Trotta, A., Falaschi, P., Cornara, L., Minganti, V., Fusconi, A., Drava, G., \& Berta, G. (2006). Arbuscular mycorrhizae increase the arsenic translocation factor 
in the As hyperaccumulating fern Pteris vittata L. Chemosphere, 65, 74-81.

Tryon, R. M., \& Tryon, A. F. (1982). Ferns and allied plants with special reference to tropical America. New York: Springer-Verlag.

Turnau, K., Anielska, T., \& Jurkiewicz, A. (2005). Mycothallic/mycorrhizal symbiosis of chlorophyllous gametophytes and sporophytes of a fern, Pellaea viridis (Forsk) Prantl. (Pellaeaceae, Pteridales). Mycorrhiza, 15, 121-128.

van der Heijden, M. G. A., Martin, F., Selosse, M. A., \& Sanders, I. R. (2015). Mycorrhizal ecology and evolution: the past, the present, and the future. New Phytologist, 205, 1406-1423.

Wang, B., \& Qiu, Y. L. (2006). Phylogenetic distribution and evolution of mycorrhizas in land plants. Mycorrhiza, 16, 299-363.

Wang, Y., Zhan, M., Zhu, H., Guo, S., Wang, W., \& Xue, B. (2012). Distribution and accumulation of metals in soils and plant from a lead-zinc mineland in Guangxi, South China. Bulletin of Environmental Contamination and Toxicology, 88, 198-203.

West, B., Brandt, J., Holstien, K., Hill, A., \& Hill, M. (2009). Fern-associated arbuscular mycorrhizal fungi are represented by multiple Glomus spp: do environmental factors influence partner identity? Mycorrhiza, 19, 295-304.

Winther, J. L., \& Friedman, W. E. (2008). Arbuscular mycorrhizal associations in Lycopodiaceae. New Phytologist, 177, 790-801.

Winther, J. L., \& Friedman, W. E. (2009). Phylogenetic affinity of arbuscular mycorrhizal symbionts in Psilotum nudum. Journal of Plant Research, 122, 485-496.

Yi, W., Fu, S., Cao, H., \& Ding, M. (1994). Vesiculararbuscular mycorrhizal associations in three forest types in the Dinghushan Biosphere Reserve, subtropical China. Proceedings of Australian Center for international Agricultural Research, 62, 41-46.

Zhang, Y., Guo, L. D, \& Liu, R. J. (2004). Arbuscular mycorrhizal fungi associated with common pteridophytes in Dujiangyan, southwest China. Mycorrhiza, $14,25-30$.

Zhao, Z. W. (2000). The arbuscular mycorrhizas of pteridophytes in Yunnan, southwestern China: evolutionary interpretations. Mycorrhiza, 10, 145-149.

Zubek, S., Piatek, K., Naks, P., Heise, W., Wayda, M., \& Mleczko, P. (2010). Fungal root endophyte colonization of fern and lycophyte species from the Celaque National Park. American Fern Journal, 100, 126-136. 